\title{
Effect of contacts on spin lifetime measurements in graphene
}

\author{
Evan Sosenko, ${ }^{*}$ Huazhou Wei, ${ }^{\dagger}$ and Vivek Aji ${ }^{\ddagger}$ \\ Department of Physics, University of California, Riverside, Riverside, California 92521, USA
}

(Dated: June 26, 2021)

\begin{abstract}
Injection, transmission, and detection of spins in a conducting channel are the basic ingredients of spintronic devices. Long spin lifetimes during transit are an important ingredient in realizing this technology. An attractive platform for this purpose is graphene, which has high mobilities and low spin-orbit coupling. Unfortunately, measured spin lifetimes are orders of magnitude smaller than theoretically expected. A source of spin loss is the resistance mismatch between the ferromagnetic electrodes and graphene. While this has been studied numerically, here we provide a closed form expression for Hanle spin precession which is the standard method of measuring spin lifetimes. This allows for a detailed characterization of the nonlocal spin valve device.
\end{abstract}

\section{INTRODUCTION}

Spintronic devices rely on the ability to inject, transport, manipulate, and detect spins [1,2]. The typical architecture involves ferromagnetic electrodes deposited on a conducting medium [3,4]. Driving a current across the junction of a magnetic element and a nonmagnetic metal leads to spin injection (also called spin accumulation) [4-7]. The injected spins either diffuse in nonlocal spin valve geometry, or are driven by applied fields across the conducting channel. The former has the advantage that the observed spin signal is not corrupted by accompanying charge current. During this transit, scattering processes dephase the spins and thus degrade the chemical potential imbalance between spins of opposite orientation. The residual difference is detected by a ferromagnetic electrode whose magnetization can be flipped by applying external fields.

The performance of devices is determined by a number of parameters associated with the basic processes described above. The efficiency of spin injection, the diffusion length (or equivalently the diffusion constant and spin relaxation time), the distance between the injector and detector, and resistivities of various components such as the electrodes, the junction, and the conducting channel, are some of the ingredients that contribute to the measured magnetoresistance. As such, having good injection efficiency coupled with long spin lifetimes is crucial for the viability of spintronic applications. The discovery of graphene [8] has been of particular interest in this regard because of its tunable conductivity, high mobility, and low spin-orbit coupling. Moreover, the two dimensional nature allows for efficient device design and spin manipulation. Theoretical estimates for spin lifetimes of a few microseconds $[9,10]$ are leading to a concerted effort in realizing spin based transistors and spin valves [11-18].

Unfortunately, the best measured spin lifetimes via the Hanle spin precession technique are in the 50 ps to $200 \mathrm{ps}$

\footnotetext{
* evan.sosenko@email.ucr.edu; http://evansosenko.com/

† hwei002@ucr.edu

‡ vivek.aji@ucr.edu
}

range [11, 18-20]. The large discrepancy is yet to be explained. The linear scaling of spin and transport lifetimes [18] suggested that the dominant scattering mechanism in the conducting channeling is of the Elliot-Yafet [21] type. Surprisingly, in the regime of small spin lifetimes ( $\sim 100 \mathrm{ps})$, Coulomb scattering was shown not to be the dominant mechanism [20]. The more important determining factor of the lifetime was found to be the nature of the interface between the magnetic electrode and the conducting channel. Tunneling contacts suppress spin relaxation, and lifetimes of $771 \mathrm{ps}$ were reported at room temperature, increasing to $1.2 \mathrm{~ns}$ at $4 \mathrm{~K}$ [22]. On the other hand, low resistance barriers lead to considerable uncertainty in the determination of the lifetimes.

Over the last few years, characterizing the nature of the spin dynamics at the interface has garnered much attention. A key contribution in this effort is the generalization of the standard theoretical approach of calculating the nonlocal magnetoresistance with and without the magnetic field. Recent efforts study the effect of including the contact resistance [19, 23], and alternatively relaxing the normally infinite boundary conditions in favor of a finite channel size [24]. The approach relies on numerically solving the Bloch equation to generate Hanle precession curves and then fitting observed data.

In this paper, we present the closed form expression for the precession curves with finite contact resistance, and analytically discuss the various parameters regimes that show qualitatively different behaviors. The fits to data reproduce the results in the literature and provide a means to understand the effect of the contacts which were previously obtained by numerical simulations.

The paper is organized as follows. In section II we provide the basic model, define the relevant parameters, and present an expression for the nonlocal resistance $R_{\mathrm{NL}}$. The primary result is given by equation (4). In section III the solution for $R_{\mathrm{NL}}$ is fitted to data. In section IV we analyze the various regimes which are determined by the diffusion length, length of the device, and the contact resistance. Section V ends with a summary of the results and future directions. 
FIG. 1. The geometry of the nonlocal spin valve analyzed in this paper is shown. There are two ferromagnetic electrodes placed on a conducting channel. Current $I$ flows into the left electrode, while the potential $V$ is measured at the right electrode. The nonlocal resistance is defined as the ratio $V / I$. For spin dependent phenomena, the relevant quantity of interest is the difference between the nonlocal resistance for the parallel and antiparallel orientations of magnetization of the two electrodes.

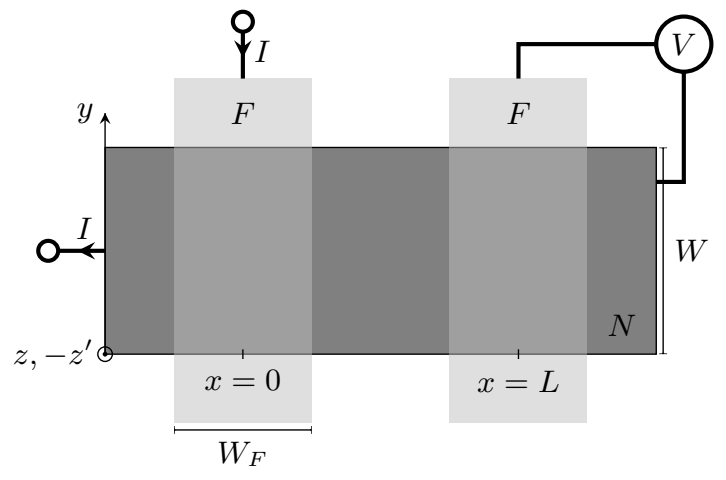

\section{MODEL}

The assumed device geometry is shown in figure 1. Two ferromagnetic contacts $(F)$ are deposited on the normal semiconductor $(N)$. A spin-polarized current $I$ is injected through the contact at $x=0$ and flows in the $x \leq 0$ region of the semiconductor. The voltage difference $V$ is measured at $x=L$ between the contact and the semiconductor. The nonlocal resistance is $R_{\mathrm{NL}}=V / I$ [23].

Spin transport is modeled by identifying two spin channels and their associated three-component spin electrochemical potentials $\mu_{\uparrow \downarrow}$. The majority channel is labeled as up, while the minority channel is labeled as down. The voltage difference is proportional to the spin accumulation $\mu_{s}=\left(\mu_{\uparrow}-\mu_{\downarrow}\right) / 2$ at $x=L$. The spin accumulation in the semiconductor is assumed to satisfy the steadystate Bloch diffusion equation

$$
D \nabla^{2} \mu_{s}^{N}-\frac{\mu_{s}^{N}}{\tau}+\omega \times \mu_{s}^{N}=0 .
$$

The key parameters are the contact spacing $L$, the diffusion constant $D$, the spin lifetime $\tau$, the spin diffusion length $\lambda=\sqrt{D \tau}$, and $\omega=\left(g \mu_{B} / \hbar\right) B$ which is proportional to the applied magnetic field $B$ and the gyromagnetic ratio $g=2$.

For contacts which cover the width of the channel, the transport is uniform along $y$. Since the channel is twodimensional, $\mu_{s}^{N}$ will only vary along $x$. We enforce the boundary condition $\mu_{s}^{N} \rightarrow 0$ at $x \rightarrow \pm \infty$ and the continuity of the current and spin current. A detailed derivation is given in appendix $\mathrm{A}$ and reveals

$$
R_{\mathrm{NL}}^{ \pm}= \pm p_{1} p_{2} R_{N} f
$$

The overall sign corresponds to parallel and antiparallel ferromagnetic alignments. Specifically, we find a resis- tance scale

$$
R_{N}=\frac{\lambda}{W L} \frac{1}{\sigma^{N}}
$$

and the function

$$
\begin{gathered}
f=\operatorname{Re}\left(\left\{2\left[\sqrt{1+i \omega \tau}+\frac{\lambda}{2}\left(\frac{1}{r_{0}}+\frac{1}{r_{L}}\right)\right] e^{(L / \lambda) \sqrt{1+i \omega \tau}}\right.\right. \\
\left.\left.+\frac{\lambda^{2}}{r_{0} r_{L}} \frac{\sinh [(L / \lambda) \sqrt{1+i \omega \tau}]}{\sqrt{1+i \omega \tau}}\right\}^{-1}\right)
\end{gathered}
$$

Note that $f$ is unitless and depends only on the scales $L / \lambda, \omega \tau$, and $\lambda / r_{i}$. The parameters $r_{i}$ with $i$ either 0 for the left contact or $L$ for the right are

$$
r_{i}=\frac{R_{F}+R_{C}^{i}}{R_{\mathrm{SQ}}} W
$$

where $R_{F}$ is the resistance of the ferromagnet and $R_{C}^{i}$ are the individual contact resistances, $W$ is the graphene flake width, and

$$
R_{\mathrm{SQ}}=W / \sigma^{N}
$$

is the graphene square (sheet) resistance given in terms of the semiconductor conductivity $\sigma^{N}$. The resistances $R_{F}$ and $R_{C}^{i}$ are the effective resistances of a unit cross sectional area. They are defined in equations (A10) and (A15). To obtain an expression in terms of the ohmic resistances, one must make the substitutions $R_{F} \rightarrow$ $W_{F} W R_{F}$ and $R_{C}^{i} \rightarrow W_{F} W R_{C}^{i}$, where $W_{F}$ is the contact width, i.e., $W_{F} W$ is the contact area. We will use the same symbols for either resistance type when the meaning is clear. The polarizations $p_{1}$ and $p_{2}$, defined in equation (A36), model the effective current injection. They depend on the resistances and the spin polarizations of the semiconductor and the individual contacts.

The expression $\Delta R_{\mathrm{NL}}=\left|R_{\mathrm{NL}}^{+}-R_{\mathrm{NL}}^{-}\right|$measures the difference in signal between parallel and antiparallel field alignments. We combine $P^{2}=\left|p_{1} p_{2}\right|$ [25], and write

$$
\Delta R_{\mathrm{NL}}=2 P^{2} R_{N}|f|
$$

with

$$
R_{N}=\frac{\lambda}{W} \frac{1}{\sigma_{G}}
$$

where $\sigma_{G}=\sigma^{N} L$ is the graphene conductance normally given in units of $\mathrm{mS}=(\mathrm{m} \Omega)^{-1}$.

\section{FITS}

Data presented in figure 4 from [26] was fit to the model presented here. Fits done using Python and matplotlib [27]. Links to the source code along with instructions on 
how to create similar fits and figures are available online [28].

We assume similar contacts, $R_{C}=R_{C}^{0}=R_{C}^{L}$. The resistance of the ferromagnet Co is computed as $R_{F}=$ $\rho_{F} \lambda_{F} / A_{J}$, where $\rho_{F}$ is the Co resistivity, $\lambda_{F}$ is the spin diffusion length of Co, and $A_{J}$ is the junction area estimated at $A_{J}=W d$, with $d$ between $0.5 \mathrm{~nm}$ and $50 \mathrm{~nm}$ [26]. Hanle fits were done using a simple least squares algorithm with nonnegative parameters $\tau, D, R_{C}$, and $P$. The polarization $P$ was constrained between zero and one.

Figure 2 shows fits of $\Delta R_{\mathrm{NL}}$ given by equation (7) for devices with tunneling and transparent contacts, and $R_{\mathrm{NL}}^{+}$given by equation (2) for a device with pinhole contacts [29]. Fits (a), (b), and (c) with tunneling and pinhole contacts give large $R_{C} \sim 10^{7} \mathrm{k} \Omega$ and lifetimes equivalent to fitting with $R_{C} \rightarrow \infty$, while (d) with transparent contacts gives a reduced $R_{C} \sim 3 \mathrm{k} \Omega$ and a lifetime increased by at most a factor of two (compare to $78 \mathrm{ps}$ for $\left.R_{C} \rightarrow \infty\right)$. For tunneling contacts, the polarization $P$ is $25 \%$ to $60 \%$ smaller than the lower bound given in [26], while for transparent contacts, $P$ is reduced by an order of magnitude.

Note that we have used $R_{C}$ as a fitting parameter. In most devices, this quantity can be experimentally determined, thus further constraining the fitting algorithm. As we will discuss further in the next section, a fact that becomes apparent from our analytic result is that the relevant scale is $\lambda / r$. Once $r$ becomes larger than $\lambda$, all of the corrections to the $R_{C} \rightarrow \infty$ limit Hanle curves become very small. In other words, once $r \gg \lambda$, the fit is insensitive to the actual value of the contact resistance. The fact that we quote a resistance of order $10^{7} \mathrm{k} \Omega$ in fits (a), (b), and (c) in figure 2 results from the built-in accuracy we demand of the fitting algorithm. A good fit can be obtained for any $r$ as long as it is larger than $\lambda$.

\section{REGIMES}

In this section we discuss the various limits of the expression describing the Hanle precession curve. First, we show that the commonly used results for zero magnetic field and tunneling contacts are correctly reproduced. Next, we discuss regimes where appropriate scaling will give non-unique Hanle fits. In the following, we consider the case $r=r_{0}=r_{L}$ of similar contacts.

In the limit of tunneling contacts, $R_{C}^{0}, R_{C}^{L} \gg R_{F}$. Putting $r_{0}, r_{L} \rightarrow \infty$ gives $p_{1} p_{2} \rightarrow\left(P_{\Sigma}^{L}\right)^{2}$ and

$$
f^{\infty}=\operatorname{Re} \frac{e^{-(L / \lambda) \sqrt{1+i \omega \tau}}}{2 \sqrt{1+i \omega \tau}}
$$

which is of the same form as found in appendix B of [30] (we will denote this limit with the superscript $\infty$ ). Fitting with this expression was found to give results
FIG. 2. Data in figure 4 from [26] fit to equation (7) or equation (2) with the following values: $W=2.2 \mu \mathrm{m}, W_{F}=$ $1.0 \mu \mathrm{m}, \sigma_{G}=0.5 \mathrm{mS}, \rho_{F}=60 \Omega \mathrm{nm}$, and $R_{F}=3.27 \Omega(d=$ $0.5 \mathrm{~nm}$ and $\left.\lambda_{F}=0.06 \mu \mathrm{m}\right)$. The contact type (tunneling, pinhole, or transparent) and the contact separation $L$ varies.
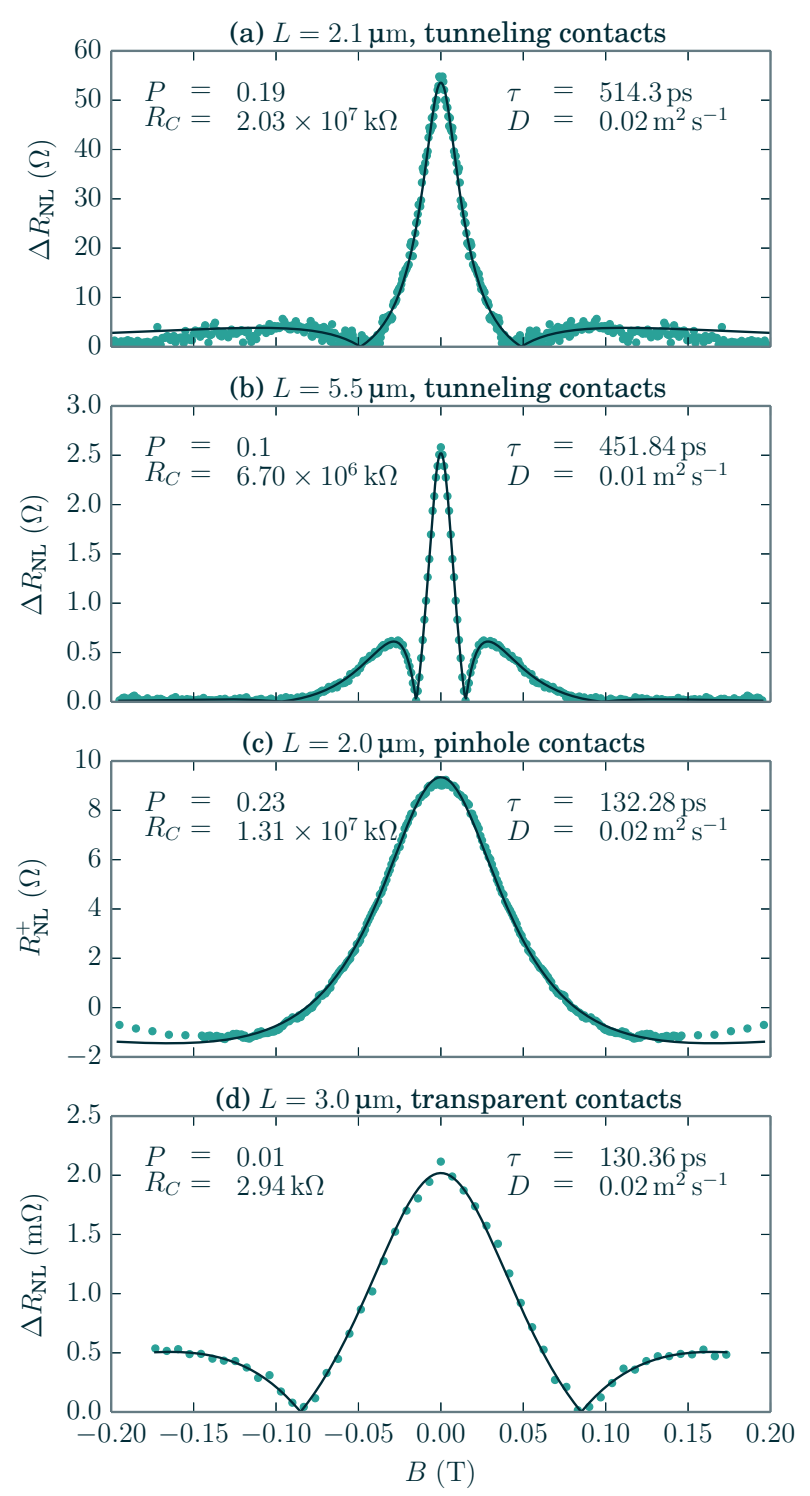

equivalent to fitting with the Hanle equation

$$
R_{\mathrm{NL}}^{ \pm}= \pm S_{\mathrm{NL}} \int_{0}^{\infty} \frac{e^{-t / \tau}}{\sqrt{4 \pi D t}} \exp \left[-\frac{L^{2}}{4 D t}\right] \cos \omega t d t .
$$

The agreement is expected as an explicit integration of equation (10) yields the same analytic expression with the identification $S_{\mathrm{NL}}=p_{1} p_{2} D / W \sigma_{G}$. In the additional 
limit of zero magnetic field,

$$
\Delta R_{\mathrm{NL}}=\left(P_{\Sigma}^{L}\right)^{2} R_{N} e^{-L / \lambda},
$$

which agrees with equation (6) in [23].

Let $f_{0}$ denote $f$ at zero magnetic field,

$$
f_{0}=\left[2(1+\lambda / r) e^{L / \lambda}+(\lambda / r)^{2} \sinh L / \lambda\right]^{-1},
$$

which agrees with equation (3) in [19].

To further explore the nature of the Hanle curves, we exploit the fact that it only depends on the dimensionless ratios $\lambda / r, L / \lambda$, and $\omega \tau$. The only other parameter of the conducting channel that enters the expression is the overall scale $\lambda$ in $R_{N}$. The expression $f$ contains three terms which are of zeroth, first, and second order in $\lambda / r$. Thus, as the contact resistance decreases, one goes from a device dominated by the first term to one dominated by the last. But precisely how this comes about depends on the value of $\omega \tau$.

For infinite contact resistance, it was pointed out that any rescaling of $g, \tau$ and $D$ that leaves $\lambda$ and $\omega \tau$ unchanged leads to the same Hanle precession curves [31]. Our result shows that the same is also true when the contact resistance is taken into account. In numerical simulations, interesting features were observed when $L / \lambda \ll 1$ and $r / \lambda \ll 1[32]$.

To compare across regimes, we first normalize the data to its value at zero magnetic field. In devices where $\lambda / r \gg 1$, the normalization factor is

$$
f_{0}=\frac{2 e^{-L / \lambda}}{(\lambda / r)^{2}}
$$

In this regime, if $D$ is not very different from the infinite contact resistance value, then the lifetime can be large, i.e., $\tau \gg 1 \mathrm{~ns}$. As one tunes the magnetic field $\sqrt{\omega \tau} \gg 1$, for small values of the field, and for much of the curve, we can approximate $1+i \omega \tau \approx i \omega \tau$. An interesting consequence of this is that the zero of the Hanle precession curve becomes independent of the scattering time. Note that the product

$$
\frac{L}{\lambda} \sqrt{\omega \tau}=L \sqrt{\frac{D}{\omega}}
$$

which appears in the exponential and oscillating factors below, is independent of the lifetime. As one further tunes the magnetic field, the Hanle curve is given by

$$
f=\frac{\sqrt{\omega \tau}}{(\lambda / r)^{2}} e^{-(L / \lambda) \sqrt{\omega \tau / 2}} \sin \left[\frac{L}{\lambda} \sqrt{\frac{\omega \tau}{2}}+\frac{\pi}{4}\right]
$$

as long as $\lambda / r \gg \sqrt{\omega \tau} \gg 1$. In this limit, the nonlocal resistance scales as

$$
\Delta R_{\mathrm{NL}} \propto \frac{\lambda \sqrt{\omega \tau}}{(\lambda / r)^{2}}=r^{2} \sqrt{\frac{\omega}{D}},
$$

and the normalized nonlocal resistance as

$$
f / f_{0} \propto \sqrt{\omega \tau} .
$$

On further increasing the field, $\sqrt{\omega \tau} \gg \lambda / r \gg 1$, we get

$$
f=\frac{1}{2 \sqrt{\omega \tau}} e^{-(L / \lambda) \sqrt{\omega \tau / 2}} \cos \left[\frac{L}{\lambda} \sqrt{\frac{\omega \tau}{2}}+\frac{\pi}{4}\right] .
$$

In this limit, the nonlocal resistance scales as

$$
\Delta R_{\mathrm{NL}} \propto \frac{\lambda}{\sqrt{\omega \tau}}=\sqrt{\frac{D}{\omega}}
$$

and the normalized nonlocal resistance as

$$
f / f_{0} \propto \frac{(\lambda / r)^{2}}{\sqrt{\omega \tau}}=D \sqrt{\frac{\tau}{\omega r^{4}}} .
$$

In the limits of equations (15) and (18), the zeros of the Hanle fit are independent of the lifetime and are determined by $D$ though the condition

$$
L \sqrt{\frac{D}{2 \omega}}+\frac{\pi}{4}=\frac{n \pi}{2},
$$

where $n=0$ for equation (15) and $n=1$ for equation (18).

Note that fitting is insensitive to $\tau$ in the limit of equation (16) or equation (19). As an example of this, figure 3 shows nearly identical fits with lifetimes that differ by four orders of magnitude. These fits were obtained by choosing large starting values for $\tau$. For figure 2 (d) and figure $3, \chi^{2} \sim 7 \times 10^{-8}$, but the $\chi^{2}$ for figure 2 (d) is $2 \%$ less than the $\chi^{2}$ for figure 3. In figure 2 (d), $\lambda / r \gg \sqrt{\omega \tau}$ and $\omega \tau \sim 1$ for most of the curve, so the approximation $1+i \omega \tau \approx i \omega \tau$ does not hold. However, figure 3 is in the limit of equation (16) for all points (save the origin). Thus, in limit of small $r$, the fitted value of $\tau$ is unreliable unless one carefully controls the fitting procedure.

The evolution of the expression for the Hanle curve is an interesting insight into the behavior of the device. Fitting data on devices with small contact resistances with the functional form applicable to infinite contact resistance yields unreliable parameters. In particular, they were numerically shown to severely underestimate the spin lifetime [32].

Further analytic progress can be made if one assumes that lifetimes as estimated with infinite contact resistance are long enough that the approximation of $\sqrt{\omega \tau} \gg$ $\lambda / r \gg 1$ is still valid for much of the data being analyzed. For this case, at infinite contact resistance, the normalized nonlocal resistance is given by

$$
\frac{f^{\infty}}{f_{0}^{\infty}}=\frac{1}{\sqrt{\omega \tau}} e^{-(L / \lambda) \sqrt{\omega \tau / 2}} \cos \left[\frac{L}{\lambda} \sqrt{\frac{\omega \tau}{2}}+\frac{\pi}{4}\right] .
$$

Provided $D$ remains constant, this will yield the same curve with finite contact resistance if

$$
\frac{1}{\tau^{\infty}}=D^{2} \frac{\tau}{r^{4}} .
$$


In other words, if we fix $\tau$ and ask what happens to the fitted value assuming infinite contact resistance as a function of decreasing $r$, equation (23) shows that it will decrease as well. For $D$ fixed, $\tau^{\infty} \propto r^{4}$. While the general trend is consistent with [32], the quantitative agreement is limited by the approximations made for analytic con-

FIG. 3. Data in figure 4 (d) from [26] fit to equation (2) with the same values as in figure 2 (d). Fits with lifetimes that differ by four orders of magnitude were obtained by using different starting values for $\tau$. These fits are otherwise similar with the exception of the lifetime, demonstrating the $\tau$-independent scaling in equation (16). The $\chi^{2}$ for figure 2 (d) is $2 \%$ less than the $\chi^{2}$ for figure 3 .

(d.1) $L=3.0 \mu \mathrm{m}$, transparent contacts

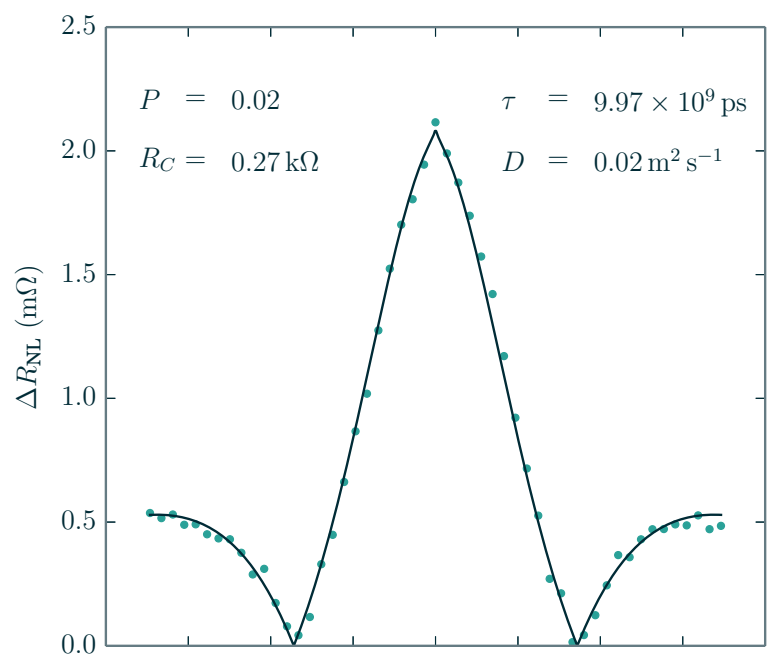

(d.2) $L=3.0 \mu \mathrm{m}$, transparent contacts

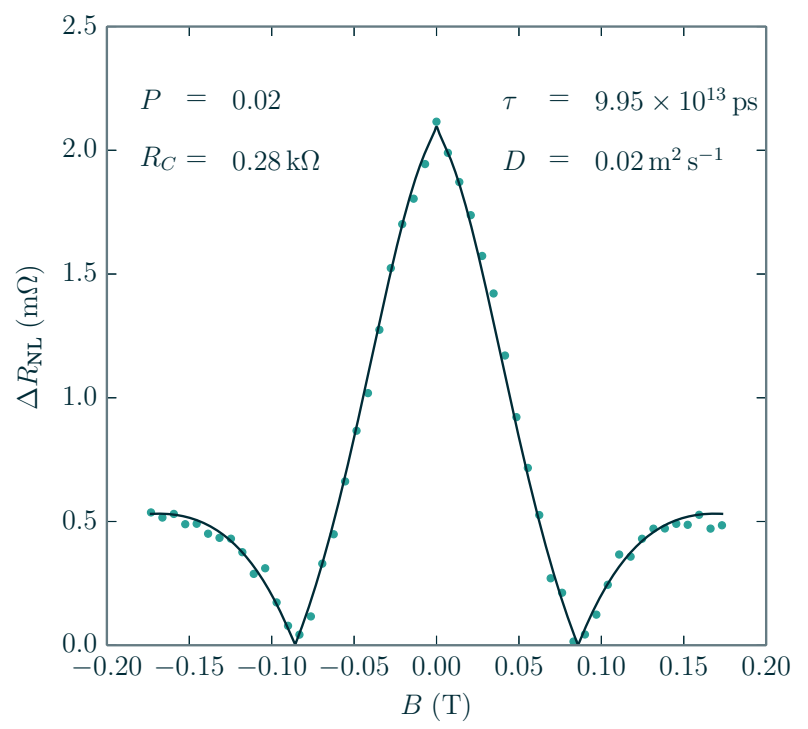

venience.

\section{SUMMARY}

In this paper we have analyzed the effect of contact resistance on spin lifetimes determined via the Hanle spin prescession technique in nonlocal spin valves. The general expression for the precession curves given in equation (4) is the main new result. While aspects of the discussed phenomena have been addressed numerically before, an analytic solution is obtained here which allows for detailed characterization of the device. In particular, general features of scaling as well as various limits and regimes can be analyzed. In addition, the solution allows for fitting data using standard curve fitting algorithms.

\section{ACKNOWLEDGMENTS}

We acknowledge useful discussions with Roland Kawakami, Adrian Swartz, and Sung-Po Chao. The work was partially supported by a UCR Senate Research Grant.

\section{Appendix A: Computation}

In this appendix we derive an expression for the nonlocal resistance for finite contact resistance. We first present the key definitions and critical boundary conditions. We then derive the relation between the nonlocal resistance and the spin chemical potential at the far contact, $\mu_{s}^{N}(L)$. Finally, we solve the diffusion equation inside the semiconductor to find $\mu_{s}^{N}(L)$.

\section{Definitions}

Many of the definitions and results in this section are taken from [33]. The chemical potential and spin chemical potential are defined in terms of the spin-up and spin-down chemical potentials,

$$
\begin{aligned}
\mu & =\frac{1}{2}\left(\mu_{\uparrow}+\mu_{\downarrow}\right), \\
\mu_{s} & =\frac{1}{2}\left(\mu_{\uparrow}-\mu_{\downarrow}\right) .
\end{aligned}
$$

The material conductances and polarization are defined in terms of the spin-up and spin-down conductances,

$$
\begin{aligned}
\sigma & =\sigma_{\uparrow}+\sigma_{\downarrow}, \\
\sigma_{s} & =\sigma_{\uparrow}-\sigma_{\downarrow}, \\
P_{\sigma} & =\frac{\sigma_{s}}{\sigma} .
\end{aligned}
$$


The gradient of the chemical potentials drives a current and spin current,

$$
\begin{aligned}
J_{\uparrow \downarrow} & =\sigma_{\uparrow \downarrow} \nabla \mu_{\uparrow \downarrow}, \\
J & =J_{\uparrow}+J_{\downarrow}=\sigma \nabla \mu+\sigma_{s} \nabla \mu_{s}, \\
J_{s} & =J_{\uparrow}-J_{\downarrow}=\sigma_{s} \nabla \mu+\sigma \nabla \mu_{s} .
\end{aligned}
$$

To indicate the material, any of the above can have a superscript $N$ (normal semiconductor) or $F$ (ferromagnet).

The contact conductances and polarization are defined in terms of the spin-up and spin-down contact conductances,

$$
\begin{aligned}
& \Sigma=\Sigma_{\uparrow}+\Sigma_{\downarrow}, \\
& \Sigma_{s}=\Sigma_{\uparrow}-\Sigma_{\downarrow}, \\
& P_{\Sigma}=\frac{\Sigma_{s}}{\Sigma} .
\end{aligned}
$$

The mismatch of the chemical potentials across the contact drives a current and spin current,

$$
\begin{aligned}
& J_{\uparrow \downarrow}^{C}=\Sigma_{\uparrow \downarrow}\left(\mu_{\uparrow \downarrow}^{N}-\mu_{\uparrow \downarrow}^{F}\right)_{c}, \\
& J^{C}=J_{\uparrow}^{C}+J_{\downarrow}^{C}, \\
& J_{s}^{C}=J_{\uparrow}^{C}-J_{\downarrow}^{C} .
\end{aligned}
$$

The subscript $c$ will always denote the function evaluated at the contact.

We will use the term current to refer to $J$, when in fact this is a particle current density. For constant $J$, the physical charge current $I$ will be related to $J$ by a relation $I=-A J / e$ for some characteristic area $A$.

To reduce the number of subscripts and superscripts in the following, we adopt the notation for the potentials

$$
\begin{array}{ll}
u=\mu_{s}^{N}, & \varphi=\mu_{s}^{F}, \\
v=\mu^{N}, & \psi=\mu^{F},
\end{array}
$$

and currents

$$
\begin{gathered}
\jmath=J_{s}, \\
J_{c}=J^{C}, \\
\jmath_{c}=J_{s}^{C} .
\end{gathered}
$$

We rewrite equation (A5) as

$$
\begin{aligned}
& J_{c}=\Sigma\left(v_{c}-\psi_{c}\right)+\Sigma_{s}\left(u_{c}-\varphi_{c}\right), \\
& J_{c}=\Sigma_{s}\left(v_{c}-\psi_{c}\right)+\Sigma\left(u_{c}-\varphi_{c}\right),
\end{aligned}
$$

and equations (A2) and (A3) as

$$
\jmath=P_{\sigma} J+4 \frac{\sigma_{\uparrow} \sigma_{\downarrow}}{\sigma} \nabla \mu_{s} .
$$

Using equations (A4) and (A7),

$$
\jmath_{c}=P_{\Sigma}^{i} J_{c}+R_{C}^{i-1}\left(u_{c}-\varphi_{c}\right),
$$

where the contact resistance is

$$
R_{C}^{i}=\frac{\Sigma^{i}}{4 \sum_{\uparrow}^{i} \Sigma_{\downarrow}^{i}}
$$

The superscript $i$ allows for contacts with difference conductances.

\section{Boundary conditions}

In this sections, we derive the relations between the potentials and the currents This corresponds to the needed boundary conditions.

\section{a. Semiconductor}

For the semiconductor, $\sigma_{\uparrow}^{N}=\sigma_{\downarrow}^{N}=\sigma^{N} / 2$, so $P_{\sigma}^{N}=0$. Evaluating equation (A8) at the contact gives

$$
\begin{gathered}
\jmath_{c}^{N}=\sigma^{N}(\nabla u)_{c} . \\
b . \quad \text { Ferromagnet }
\end{gathered}
$$

For the ferromagnet, one assumes $\mu_{s}^{F}$ satisfies the one dimensional diffusion equation. We choose the $z^{\prime}$ coordinate antiparallel to $z$ with origin at the contact. The equation

$$
\varphi^{\prime \prime}\left(z^{\prime}\right)-k_{F}^{2} \varphi\left(z^{\prime}\right)=0,
$$

with the boundary condition $\lim _{z^{\prime} \rightarrow-\infty} \varphi\left(z^{\prime}\right)=0$ has solution

$$
\varphi\left(z^{\prime}\right)=\varphi_{c} e^{k_{F} z^{\prime}},
$$

where $\varphi_{c}=\varphi(0)$ is a yet undetermined constant. Putting this into equation (A8) and evaluating it at the contact gives

$$
\jmath_{c}^{F}=P_{\sigma}^{F} J_{c}^{F}+R_{F}^{-1} \varphi_{c},
$$

where the ferromagnet resistance is

$$
R_{F}=\frac{\sigma^{F}}{4 \sigma_{\uparrow}^{F} \sigma_{\downarrow}^{F} k_{F}} .
$$

Here, $\lambda_{F}=1 / k_{F}$ is the spin diffusion length in the ferromagnet.

\section{c. Continuity assumptions}

At the contact, the current and spin current are assumed continuous,

$$
\begin{aligned}
& J_{c}=J_{c}^{F}=J_{c}^{N}, \\
& \jmath_{c}=J_{c}^{F}=\jmath_{c}^{N} .
\end{aligned}
$$

Using equations (A9), (A14), and (A16) we find the relation

$$
\left(P_{\sigma}^{F} R_{F}+P_{\Sigma}^{i} R_{C}^{i}\right) J_{c}=\left(R_{F}+R_{C}^{i}\right) \jmath_{c}-u_{c},
$$

and that $\varphi_{c}$ is determined by

$$
R_{F}^{-1} \varphi_{c}=\frac{\left(P_{\Sigma}^{i}-P_{\sigma}^{F}\right) R_{C}^{i} \jmath_{c}+P_{\sigma}^{F} u_{c}}{P_{\sigma}^{F} R_{F}+P_{\Sigma}^{i} R_{C}^{i}} .
$$


In the special case of zero current at the contact $\left(J_{c}=0\right)$, equation (A17) reduces to

$$
\begin{aligned}
& \jmath_{c}=\frac{1}{R_{F}+R_{C}^{i}} u_{c}, \\
& \varphi_{c}=\frac{R_{F}}{R_{F}+R_{C}^{i}} u_{c} .
\end{aligned}
$$

\section{Nonlocal resistance}

In this section we derive the precise relation between $R_{\mathrm{NL}}$ and $\mu_{s}^{N}(L)$. Note that we may write in general, for some $\bar{\mu}$,

$$
\mu=\bar{\mu}+P_{\sigma} \mu_{s},
$$

and, following [23], define the voltage due to the difference in the chemical potentials across the contacts by

$$
V_{c}=\left(\bar{\mu}_{c}^{N}-\bar{\mu}_{c}^{F}\right) / e .
$$

We assume a fixed current $J_{0}=\left|J_{0}\right|>0$ flows down through the contact at $x=0$ and to the left in the semiconductor for $x \leq 0$, and no current flows for $x>0$. The experimentally measured quantity is the nonlocal resistance $R_{\mathrm{NL}}=V_{L} / I_{0}$, where $I_{0}=-W L J_{0} / e$ is the current through the contact at $x=0$. It is convenient to introduce the effective nonlocal resistance $R_{\mathrm{NL}}^{\mathrm{SQ}}$ defined by

$$
R_{\mathrm{NL}}^{\mathrm{SQ}}=W L R_{\mathrm{NL}}=-e V_{L} / J_{0}=\frac{\bar{\mu}_{c}^{F}-\bar{\mu}_{c}^{N}}{J_{0}} .
$$

To determine $R_{\mathrm{NL}}$, we must express the difference of these chemical potentials in terms of $\mu_{s}^{N}(L)$.

Since there are two ferromagnetic contacts, we have separate functions $\psi$ and $\varphi$ for each contact which we will denote by $\psi^{0}, \varphi^{0}$, and $\psi^{L}, \varphi^{L}$. From equation (A13), we have

$$
\begin{aligned}
\varphi^{0}\left(z^{\prime}\right) & =\varphi_{0} e^{k_{F} z^{\prime}}, \\
\varphi^{L}\left(z^{\prime}\right) & =\varphi_{L} e^{k_{F} z^{\prime}} .
\end{aligned}
$$

The physical restriction on the current flow in the semiconductor is imposed by noting that since $\sigma_{s}^{N}=0$, equation (A3b) gives $J^{N}=\sigma^{N} \nabla v$, so we must have

$$
v_{x}(x)= \begin{cases}v_{x}(0)-\left(J_{0} / \sigma^{N}\right) x & \text { for } x \leq 0 \\ v_{x}(0) & \text { for } x>0\end{cases}
$$

$v_{y}(x)=v_{y}(0)$, and $v_{z}(x)=v_{z}(0)$.

Using equation (A3b), the restriction on the current flow in each ferromagnet gives

$$
\begin{aligned}
\nabla \psi^{0} & =\left(J_{0} / \sigma^{F}\right)-P_{\sigma}^{F} \nabla \varphi^{0}, \\
\nabla \psi^{L} & =-P_{\sigma}^{F} \nabla \varphi^{L} .
\end{aligned}
$$

Integrating and enforcing $e V_{c}=v_{x}(0)-\left(\psi_{c}-P_{\sigma}^{F} \varphi_{c}\right)$,

$$
\begin{aligned}
\psi^{0}\left(z^{\prime}\right)=- & e V_{0}+P_{\sigma}^{F} \varphi_{0}\left(2-e^{k_{F} z^{\prime}}\right) \\
& +v_{x}(0)+\left(J_{0} / \sigma^{F}\right) z^{\prime}, \\
\psi^{L}\left(z^{\prime}\right)=- & e V_{L}+P_{\sigma}^{F} \varphi_{L}\left(2-e^{k_{F} z^{\prime}}\right) \\
& +v_{x}(0) .
\end{aligned}
$$

There is no current at the contact at $x=0$, thus equation (A7a) gives

$$
\psi_{L}-v_{L}=P_{\Sigma}^{L}\left(u_{L}-\varphi_{L}\right),
$$

and with equation (A18b), we find

$$
\begin{aligned}
R_{\mathrm{NL}}^{\mathrm{SQ}} & =\left(\psi_{L}-v_{L}\right)-P_{\sigma}^{F} \varphi_{L} \\
& =\left[P_{\Sigma}^{L}\left(1-\frac{R_{F}}{R_{F}+R_{C}^{L}}\right)-\frac{P_{\sigma}^{F} R_{F}}{R_{F}+R_{C}^{L}}\right] \frac{u_{x}(L)}{J_{0}} .
\end{aligned}
$$

\section{Diffusion equation}

In this section we show how to solve for $\mu_{s}^{N}(L)$. This method is based on the one described in [19]. Inside the semiconductor, $u$ satisfies the diffusion equation

$$
D \nabla^{2} u-\frac{u}{\tau}+\omega \times u=0 .
$$

Here, $D$ is the diffusion constant, $\tau$ the spin lifetime, and $\omega=\left(g \mu_{B} / \hbar\right) B$ is proportional to the applied magnetic field (with $g$ the gyromagnetic ratio and $\mu_{B}$ the Bohr magneton). The spin diffusion length in the semiconductor is $\lambda=1 / k=\sqrt{D \tau}$.

The function $u=u(x)$ only varies along $x$, and we introduce the notation

$$
u_{x}(x)=\left\{\begin{array}{l}
u_{x-}(x) \text { for } x<0, \\
u_{x 0}(x) \text { for } 0 \leq x \leq L, \\
u_{x+}(x) \text { for } L<x
\end{array}\right.
$$

with similar expressions for $u_{y}$ and $u_{z}$. The most general solution to equation (A28) decouples $u_{z}$ from $u_{x}$ and $u_{y}$. The requirement $\lim _{x \rightarrow \pm \infty} u(x)=0$ yields

$$
\begin{aligned}
& u_{z \pm}(x)=A^{\mp} e^{\mp k x}, \\
& u_{z 0}(x)=A_{0}^{+} e^{k x}+A_{0}^{-} e^{-k x},
\end{aligned}
$$

and

$$
\begin{gathered}
u_{x \pm}(x)=B^{\mp} e^{\mp \kappa x}+C^{\mp} e^{\mp \bar{\kappa} x}, \\
u_{y \pm}(x)=i B^{\mp} e^{\mp \kappa x}-i C^{\mp} e^{\mp \bar{\kappa} x}, \\
u_{x 0}(x)=B_{0}^{+} e^{\kappa x}+B_{0}^{-} e^{-\kappa x}+C_{0}^{+} e^{\bar{\kappa} x}+C_{0}^{-} e^{-\bar{\kappa} x}, \\
u_{y 0}(x)=i B_{0}^{+} e^{\kappa x}+i B_{0}^{-} e^{-\kappa x}-i C_{0}^{+} e^{\bar{\kappa} x}-i C_{0}^{-} e^{-\bar{\kappa} x},
\end{gathered}
$$


where $\kappa=k \sqrt{1+i \omega \tau}$. The twelve constants $A, B$ and $C$ (with their various subscripts and superscripts) must be determined by imposing the appropriate boundary conditions.

We first require $u$ be continuous at $x=0$ and $x=L$; this gives six equations. We now require a boundary condition on $\nabla u$, but $\nabla u$ cannot be assumed continuous at the contact. We make the assumption that the total spin current at the contact is the sum of the spin currents on either side, i.e.,

$$
\begin{aligned}
& \jmath_{0}=\sigma^{N}\left[-u_{-}^{\prime}(0)+u_{0}^{\prime}(0)\right], \\
& \jmath_{L}=\sigma^{N}\left[-u_{0}^{\prime}(L)+u_{+}^{\prime}(L)\right] .
\end{aligned}
$$

The signs have been chosen to be consistent with the physical geometry. The only nonzero component of the current at the contacts inside the semiconductor is the $x$ component at $x=0$, so we use equation (A17a). For all other components there is zero current at the contact, and we use equation (A18a). Together with equation (A32), this gives the other six equations,

$$
\begin{aligned}
-u_{z-}^{\prime}(0)+u_{z 0}^{\prime}(0)+\eta_{0} u_{z}(0) & =0, \\
u_{z+}^{\prime}(L)-u_{z 0}^{\prime}(L)+\eta_{L} u_{z}(L) & =0, \\
-u_{x-}^{\prime}(0)+u_{x 0}^{\prime}(0)+\eta_{0} u_{x}(0) & =\Delta, \\
u_{x+}^{\prime}(L)-u_{x 0}^{\prime}(L)+\eta_{L} u_{x}(L) & =0, \\
-u_{y-}^{\prime}(0)+u_{y 0}^{\prime}(0)+\eta_{0} u_{y}(0) & =0, \\
u_{y+}^{\prime}(L)-u_{y 0}^{\prime}(L)+\eta_{L} u_{y}(L) & =0,
\end{aligned}
$$

where

$$
\begin{aligned}
\eta_{i}^{-1} & =-\sigma^{N}\left(R_{F}+R_{C}^{i}\right), \\
\Delta & =-\left(-J_{0}\right)\left(P_{\sigma}^{F} R_{F}+P_{\Sigma}^{0} R_{C}^{0}\right) \eta_{0} .
\end{aligned}
$$

We define the $r$-parameter, $r_{i}=-\eta_{i}^{-1}$, introduced in equation (5).

These equations can be organized into a matrix equation and solved algebraically. A solution for $u_{z}$ corresponds to a condition of vanishing determinant,

$$
e^{-2 L / \lambda}=\left(1+\frac{2 r_{0}}{\lambda}\right)\left(1+\frac{2 r_{L}}{\lambda}\right)
$$

which can never be satisfied [34], thus $u_{z}=0$ is the only allowed solution. The other two components form an eight dimensional linear system. Solving this gives the remaining constants, and thus $u_{x}(L)=e^{-\kappa L} B^{-}+$ $e^{-\bar{\kappa} L} C^{-}$.

Finally, by using $p_{1}=-\sigma^{N} \Delta / J_{0}$ along with equation (A27), we can introduce $R_{\mathrm{SQ}}$ from equation (6) and the polarizations

$$
\begin{aligned}
p_{1} & =\frac{P_{\sigma}^{F} R_{F}+P_{\Sigma}^{L} R_{C}^{L}}{R_{F}+R_{C}^{L}}, \\
p_{2} / p_{1} & =\left(1-\frac{P_{\sigma}^{F} R_{F}}{P_{\Sigma}^{L} R_{C}^{L}}\right) /\left(1+\frac{P_{\sigma}^{F} R_{F}}{P_{\Sigma}^{L} R_{C}^{L}}\right),
\end{aligned}
$$

to write

$$
\frac{R_{\mathrm{NL}}^{\mathrm{SQ}}}{R_{\mathrm{SQ}}}=\frac{p_{1} p_{2}}{W / \lambda}\left[-\frac{k u_{x}(L)}{\Delta}\right] .
$$

The factor in brackets is the function $f$ given in equation (4).
[1] S. A. Wolf, D. D. Awschalom, R. A. Buhrman, J. M. Daughton, S. von Molnár, M. L. Roukes, A. Y. Chtchelkanova, and D. M. Treger, Science 294, 1488 (2001).

[2] I. Žutić, J. Fabian, and S. Das Sarma, Rev. Mod. Phys. 76, 323 (2004).

[3] S. Datta and B. Das, Applied Physics Letters 56, 665 (1990).

[4] F. J. Jedema, A. T. Filip, and B. J. van Wees, Nature 410, 345 (2001).

[5] M. Johnson and R. H. Silsbee, Phys. Rev. Lett. 55, 1790 (1985).

[6] T. Yang, T. Kimura, and Y. Otani, Nat Phys 4, 851 (2008).

[7] S. O. Valenzuela, D. J. Monsma, C. M. Marcus, V. Narayanamurti, and M. Tinkham, Phys. Rev. Lett. 94, 196601 (2005).

[8] K. S. Novoselov, A. K. Geim, S. V. Morozov, D. Jiang, Y. Zhang, S. V. Dubonos, I. V. Grigorieva, and A. A. Firsov, Science 306, 666 (2004).

[9] D. Huertas-Hernando, F. Guinea, and A. Brataas, Phys.
Rev. B 74, 155426 (2006).

[10] B. Trauzettel, D. V. Bulaev, D. Loss, and G. Burkard, Nat Phys 3, 192 (2007).

[11] N. Tombros, C. Jozsa, M. Popinciuc, H. T. Jonkman, and B. J. van Wees, Nature 448, 571 (2007).

[12] M. Ohishi, M. Shiraishi, R. Nouchi, T. Nozaki, T. Shinjo, and Y. Suzuki, Japanese Journal of Applied Physics 46, L605 (2007).

[13] S. Cho, Y.-F. Chen, and M. S. Fuhrer, Applied Physics Letters 91, 123105 (2007).

[14] N. Tombros, S. Tanabe, A. Veligura, C. Jozsa, M. Popinciuc, H. T. Jonkman, and B. J. van Wees, Phys. Rev. Lett. 101, 046601 (2008).

[15] E. Hill, A. Geim, K. Novoselov, F. Schedin, and P. Blake, Magnetics, IEEE Transactions on 42, 2694 (2006).

[16] W. Han, J.-R. Chen, D. Wang, K. M. McCreary, H. Wen, A. G. Swartz, J. Shi, and R. K. Kawakami, Nano Letters 12, 3443-3447 (2012).

[17] W. Han, K. McCreary, K. Pi, W. Wang, Y. Li, H. Wen, J. Chen, and R. Kawakami, Journal of Magnetism and Magnetic Materials 324, 369 (2012). 
[18] C. Józsa, T. Maassen, M. Popinciuc, P. J. Zomer, A. Veligura, H. T. Jonkman, and B. J. van Wees, Phys. Rev. B 80, 241403 (2009).

[19] M. Popinciuc, C. Józsa, P. J. Zomer, N. Tombros, A. Veligura, H. T. Jonkman, and B. J. van Wees, Phys. Rev. B 80, 214427 (2009).

[20] K. Pi, W. Han, K. M. McCreary, A. G. Swartz, Y. Li, and R. K. Kawakami, Phys. Rev. Lett. 104, 187201 (2010).

[21] R. J. Elliott, Phys. Rev. 96, 266 (1954).

[22] W. Han and R. K. Kawakami, Phys. Rev. Lett. 107, 047207 (2011).

[23] S. Takahashi and S. Maekawa, Phys. Rev. B 67, 052409 (2003).

[24] M. Wojtaszek, I. J. Vera-Marun, and B. J. van Wees, (2014), arXiv:1404.6276v1 [cond-mat.mes-hall].

[25] Assuming the polarizations $P_{\sigma}^{F}$ and $P_{\Sigma}^{L}$ have the same sign bounds $P \leq 1$.

[26] W. Han, K. Pi, K. M. McCreary, Y. Li, J. J. I. Wong, A. G. Swartz, and R. K. Kawakami, Phys. Rev. Lett.
105, 167202 (2010).

[27] J. D. Hunter, Computing In Science \& Engineering 9, 90 (2007).

[28] An online portal with links to the code used to prepare this work is located at evansosenko.com/spin-lifetime/.

[29] Parallel and antiparallel data for this device was only available at dissimilar field values, thus $\Delta R_{\mathrm{NL}}$ could not be fit.

[30] M. Johnson and R. H. Silsbee, Phys. Rev. B 37, 5312 (1988).

[31] A. G. Swartz, K. M. McCreary, W. Han, H. Wen, and R. K. Kawakami, "A systematic approach to interpreting hanle spin precession data in non-local spin valves," (2013).

[32] T. Maassen, I. J. Vera-Marun, M. H. D. Guimarães, and B. J. van Wees, Phys. Rev. B 86, 235408 (2012).

[33] J. Fabian, A. Matos-Abiague, C. Ertler, P. Stano, and I. Žutić, Acta Physica Slovaca 57, 565 (2007).

[34] Except at the nonphysical point $L / \lambda=r_{i} / \lambda=0$. 
This figure "nonlocal_spin_valve.png" is available in "png" format from: http://arxiv.org/ps/1404.3211v2 